\title{
Perfil epidemiológico del donante de tejido corneal en el Hospital General de Cholula
}

\author{
Epidemiological profile of the donor of corneal \\ tissue in the General Hospital of Cholula
}

\author{
Guadalupe Merino-Cabrera, ${ }^{*, \ddagger}$ Denhi Benn Monter-Valera, ${ }^{\S}$ \\ Marcela Tejeda-Mondragón," Martha Elena Barrientos-Núñez \\ * Facultad de Medicina, Benemérita Universidad Autónoma de Puebla. Puebla, Puebla. \\ ‡ Unidad de Trasplantes Puebla. Puebla, México. \\ $\S$ Jefatura de Enseñanza, Hospital General de Cholula, Puebla. \\ " Banco de Tejido Ocular, Hospital General de Cholula, Puebla.
}

\section{RESUMEN}

Objetivo: Describir y detallar el perfil epidemiológico de los donantes de córneas en el Banco de Ojos del Hospital General de Cholula. Además de conocer los criterios de obtención, selección e inclusión de donantes, conocer las características epidemiológicas que repercuten en la viabilidad del tejido corneal con fines de trasplante. Material y métodos: En el Banco de Ojos del Hospital General de Cholula se elaboró el estudio descriptivo, transversal, observacional, incluyó la revisión de la base de datos con la información de los donantes de tejido corneal registrados durante el periodo comprendido entre junio-noviembre del año 2019. Se tuvieron en cuenta las siguientes variables: edad, sexo, serología, hospital generador, comorbilidades y causa de muerte del donante. Resultados: Se observó que de los 33 donantes registrados en la base de datos, $19(58 \%)$ fueron hombres, mientras que sólo $14(42 \%)$ fueron mujeres. Se estableció que la edad media del donante de tejido corneal en el Hospital General de Cholula fue de 58 años. Tras el análisis porcentual, la causa más frecuente de muerte en el donante fueron las enfermedades cerebrovasculares, específicamente el EVC, representando 10 casos $(31 \%)$ de los 33 donantes. La hipertensión arterial sistémica encabezó la lista de comorbilidades presentes en nuestra muestra. En cuanto al destino final de los tejidos, se determinó que el $82 \%$ logró ser trasplantado con finalidad

\begin{abstract}
Objective: To describe and detail the epidemiological profile of corneal donors in the Eye Bank of the General Hospital of Cholula. In addition to knowing the criteria for obtaining, selecting and including donors, knowing the epidemiological characteristics that affect the viability of corneal tissue for transplant purposes. Material and methods: In the Eye Bank of the General Hospital of Cholula, the descriptive, crosssectional, observational study was carried out, including the revision of the database with the information of the corneal tissue donors registered during the period between June-November of year 2019. The following variables were taken into account: age, sex, serology, generating hospital, comorbidities and cause of death of the donor. Results: It was observed that of the 33 donors registered in the database, 14 (42\%) correspond to feminine donors and 19 (58\%) to the masculine gender. The average age of the corneal tissue donor at the Cholula General Hospital was established to be 58 years. After the percentage analysis, the most frequent cause of death in the donor was cerebrovascular diseases, specifically CVD in 10 (31\%) of cases. Systemic arterial hypertension led the way in terms of comorbidities within the donor population. The number of tissues analyzed by the generator hospital during the mentioned period was recorded, leaving the General Hospital Zone 20 first with the highest percentage of productivity. Regarding the final destination of the
\end{abstract}

Citar como: Merino-Cabrera G, Monter-Valera DB, Tejeda-Mondragón M, Barrientos-Núñez ME. Perfil epidemiológico del donante de tejido corneal en el Hospital General de Cholula. Rev Mex Traspl. 2021; 10 (1): 23-28. https://dx.doi.org/10.35366/99848 
óptica. Conclusión: El perfil epidemiológico que caracterizó al donante de tejido corneal en Banco de Tejido Ocular del Hospital General de Cholula fue donante de género masculino, entre las edades 50 y 80 años, con hipertensión arterial sistémica, la causa de muerte más frecuente fueron los eventos cerebrales vasculares.

Palabras clave: Tejido corneal, trasplante, banco de ojos, donación, características epidemiológicas.

\section{INTRODUCCIÓN}

Se estima que la prevalencia nacional de ceguera en México varía del 0.4 al 1.5\%, y el $2.4-7.0 \%$ de la población tiene discapacidad visual. ${ }^{1,2}$ El amplio rango en las tasas de prevalencia se puede atribuir al número limitado de estudios epidemiológicos oftálmicos disponibles en México. En nuestro medio se estima que hay una necesidad anual de entre seis y siete mil córneas al año en enfermos que por diversos padecimientos requieren de un trasplante corneal; sin embargo, solamente se realizan alrededor de 800 , lo que deja un gran número de casos en lista de espera. ${ }^{1}$

En la actualidad, la demanda de corneas con fines ópticos supera la disponibilidad de estos tejidos y continúa siendo uno de los más requeridos. De acuerdo con los datos consultados en el sistema informático del Registro Nacional de Trasplantes, la lista de espera de receptores de córnea en enero del año 2020 fue de 5,713 pacientes.

A nivel estatal, Puebla se encuentra en sexto lugar en trasplante corneal, con un total de 169 procedimientos realizados durante el año 2019 y hasta el 03 de enero del 2020. ${ }^{3}$

El trasplante corneal es la intervención mediante la cual se sustituye tejido corneal dañado o enfermo por tejido corneal sano procedente de un donante. ${ }^{4}$ Los botones corneales pueden provenir tanto de donantes fallecidos por parada cardiaca como por muerte encefálica. Se considera que un tejido obtenido mediante procuración cadavérica es viable para trasplante cuando tiene una densidad de células endoteliales mayor de 2,000 células por milímetro. ${ }^{4,5}$

Para el proceso de procuración, existen dos técnicas de procuración de tejido corneal: la primera consiste en realizar una enucleación con la posterior escisión quirúrgica del botón corneoescleral en el Banco de Ojos; y la segunda consiste en la escisión quirúrgica del botón corneoescleral in situ. ${ }^{5}$ Esta técnica permite la colocación más temprana en medio de la preservación del tejido corneal, impone menos traumas al tejido tissues, it was determined that $82 \%$ managed to be transplanted successfully. Conclusion: The epidemiological profile that characterized the corneal tissue donor in the eye tissue bank of the General Hospital of Cholula were male donors, between the ages of 50 and 80, with systemic arterial hypertension, being the most frequent cause of death were the vascular brain events.

Keywords: Corneal tissue, transplantation, eye bank, donation, epidemiological characteristics.

corneoescleral y provee una mayor aceptación de los familiares de la donación.

Entre las funciones que de forma obligatoria se desarrollan en los bancos de ojos está realizar un control y registro casuístico de los datos generales del donante, guardar adecuada y éticamente todo lo concerniente a la causa de muerte, enfermedades acompañantes, pruebas serológicas. Es decir, se encarga del control de calidad de todos los tejidos donados, resguardo y disponibilidad de los mismos.

Partiendo de la idea anterior, es que se ha hecho hincapié en el conocimiento del perfil del donante y el cumplimiento adecuado de los protocolos en la detección y evaluación de los mismos, pues de manera indirecta hay factores que muchas veces pueden ser pasados por alto, olvidando que tienen un impacto indirecto en la calidad del tejido con fines de trasplante.

El estado de Puebla cuenta con el primer Banco de Tejido Ocular estatal, lo que ha permitido un avance de gran impacto no sólo para los programas de donación del estado, sino en la calidad de los tejidos recibidos y analizados de manera minuciosa e integral que se ha reflejado en un aumento progresivo en la demanda de córneas trasplantadas con un margen de mayor seguridad y calidad gracias a las herramientas con las que cuenta este Banco de Ojos, además de la excelente organización y logística con la que es dirigido por parte del equipo responsable.

Por eso, debemos considerar que el perfil tanto clínico como epidemiológico de los potenciales donadores de córnea varía de acuerdo con el espacio, el tiempo y las características específicas de la población en consecuencia de diversos factores, que serán analizados en el presente estudio.

\section{MATERIAL Y MÉTODOS}

En cuanto a las consideraciones éticas, este protocoló de investigación fue presentado y aprobado previamente ante el comité de bioética, así como por la 
jefatura de enseñanza del Hospital General de Cholula. Se respetaron los principios bioéticos y la normatividad establecida por el Banco de Tejido Ocular de dicho hospital. Confidencialidad de los datos. Se siguieron los protocolos del centro de trabajo sobre la publicación de datos de los donantes. ${ }^{6}$

Previa aprobación ética por parte del comité de bioética del Hospital General de Cholula, se elaboró el estudio descriptivo y transversal. Se incluyó la revisión de la base de datos con la información de los donantes de tejido corneal registrados en dicho banco durante el periodo comprendido entre junio-noviembre del año 2019, lo que concibió como muestra 61 tejidos corneales recibidos y analizados procedentes de 33 donantes con las siguientes variables consideradas: género, edad, comorbilidades, serología, diagnóstico de defunción y hospital generador.

Se excluyeron aquéllos que no se encontraran en el rango de edad aceptada para la donación de tejido corneal (2-80 años), así como donantes que contaran con antecedentes de cirugía intraocular, resultados de las pruebas de serología positivos y en quienes se desconociera la causa de muerte.

Los resultados se resumieron en tablas y gráficos, expresados en porcentajes. Durante este estudio se respetaron los principios éticos que rigen los programas de donación y trasplante en el país, así como las normas intrahospitalarias y del Banco de Tejido Ocular del Hospital General de Cholula.

\section{RESULTADOS}

En el estudio se observó que de los 33 donantes $(100 \%)$ registrados, 19 (58\%) corresponden al género masculino mientras que sólo 14 (42\%).

El donante de menor edad fue una joven de $15 \mathrm{y}$ el más longevo, un hombre de 78 años. La media de edad fue de 58 años.

Como se representa en la Figura 1, no hay registro alguno de donantes en la primera década de la vida, confirmando que las edades menores de dos años y edades tempranas no son consideradas como la mejor opción viable para trasplante. Se muestra un aumento importante en cuanto a la segunda década con el reporte de siete donadores. Para la cuarta y quinta década de la vida hay una marcada disminución, pues sólo hubo dos donantes respectivamente en esos rangos. La mayoría de la población donante considerada para este estudio perteneció a la sexta década de la vida con un total de ocho personas registradas en la base de datos de dicho Banco de Tejido.

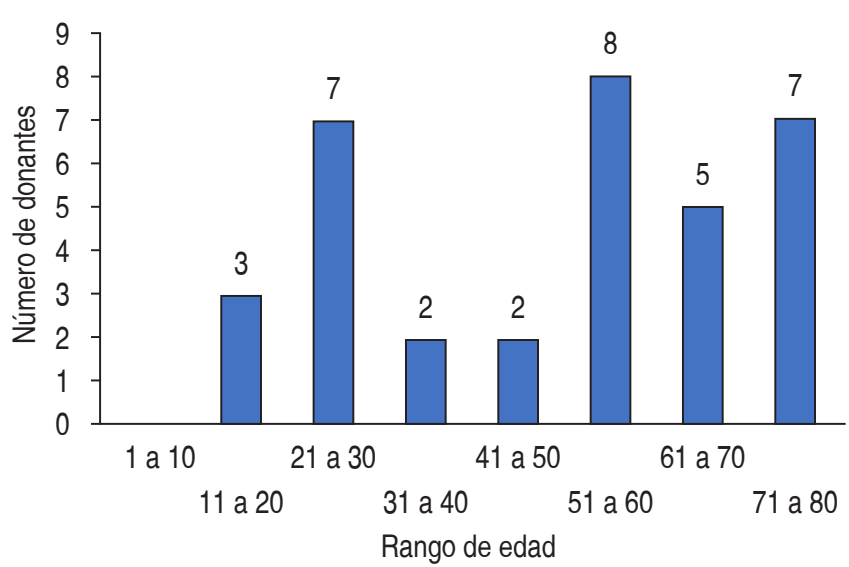

Figura 1: Representación de las edades y los rangos establecidos. Elaboración propia.

Las principales causas de mortalidad general se codificaron de acuerdo con la Clasificación Estadística Internacional de Enfermedades y Problemas Relacionados con la Salud, Décima Revisión (CIE-10) y se agruparon de la siguiente manera: alteraciones metabólicas (E70-E90), enfermedades isquémicas del corazón (I20-I25), enfermedades cerebrovasculares (I60-169), enfermedades del aparato respiratorio (J00J06) y otras afecciones cerebrales (muerte encefálica G98). Ninguno de los diagnósticos de defunción violó los criterios de selección relativos ni absolutos establecidos en el manual de procedimientos para la operación del Banco de Ojos en el Hospital General de Cholula.

Tras el análisis porcentual, se encontró que la causa más frecuente de muerte en el donante fueron las enfermedades cerebrovasculares, específicamente el EVC en 10 casos (31\%) registrados (Figura 2), dejando como la segunda causa más frecuente las enfermedades isquémicas cardiacas, presentes en 8.9 casos (27\%) de la población donante, relacionado con la epidemiología de las enfermedades crónicas degenerativas cada vez más frecuentes en nuestro país, mismas que conllevan a desarrollar alteraciones metabólicas graves que aumentan el índice de mortalidad en nuestra población, como lo fue en el caso de los cinco donadores (18\%) que fallecieron por esta causa.

Aunque sabemos que los donantes ideales serían los que desarrollan muerte encefálica, continúa siendo una causa en menor proporción epidemiológica, pues sólo hubo cinco casos (15\%). Dejando en último lugar a las enfermedades de aparato respiratorio, presentes en 2.9 donantes, es decir $9 \%$ de la población donante total. ${ }^{7-10}$ 
En cuanto al análisis de las comorbilidades adyacentes a los diagnósticos de defunción, encontramos que la mayoría de los donantes eran hipertensos, con un total de 15.8 casos, representando un $48 \%$, como se aprecia en la Figura 3, ubicando en segundo lugar con 11.8 casos (36\%) al donante diabético y en menor porcentaje aquéllos con enfermedad renal crónica, siendo la minoría con cinco pacientes (16\%).

Además de la revisión del expediente perteneciente a la base de datos del Banco de Ojos, se realizó también análisis de los botones corneales procedentes de los donantes, para determinar el destino final del tejido; sin embargo, tras la revisión y conteo celular con el apoyo de las herramientas antes mencionadas, sólo seis botones corneales (18\%) no obtuvieron la celularidad requerida ni las condiciones adecuadas para poder ser trasplantas con finalidad óptica, por lo que fueron entregadas al Departamento de Anatomía Patológica para su descarte, tal como se especifica en el manual de procedimientos para el establecimiento y operación del banco.

Sin embargo, 27 (82\%) de las 61 corneas analizadas contaron con las características adecuadas para ser trasplantadas, lo que apoya la visión y misión que tiene el Banco de Tejido Ocular en el Hospital General de Cholula, pues refleja el trabajo realizado que hay detrás de este proyecto al implementar el proceso de análisis del tejido corneal con el mayor número de recursos posibles académica y tecnológicamente que aseguren la disponibilidad de tejidos de alta calidad.

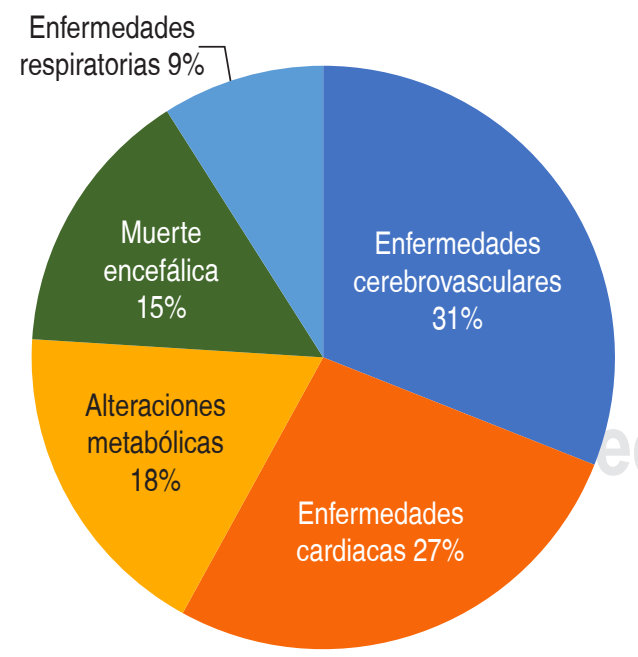

Figura 2: Diagnósticos de defunción de la población donante de tejido corneal. Elaboración propia.

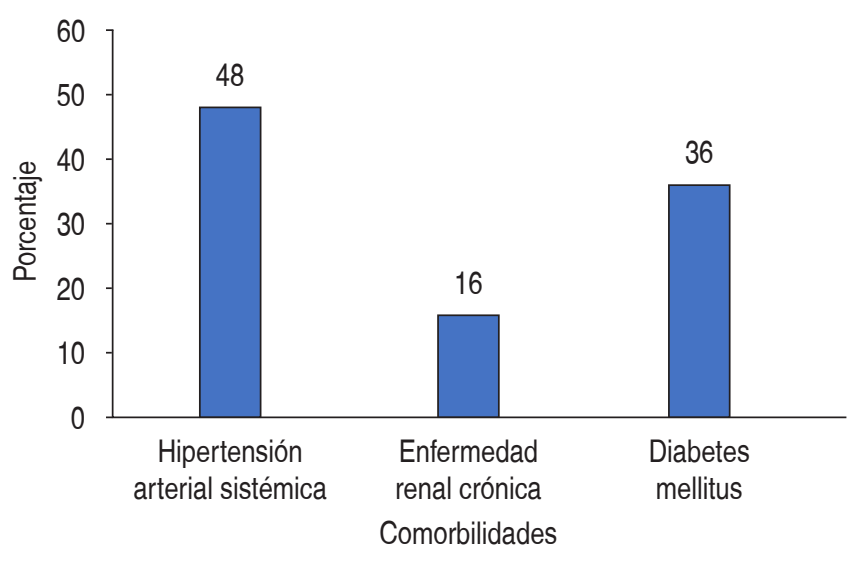

Figura 3: Comorbilidades presentes en la población donante. Elaboración propia.

\section{DISCUSIÓN}

Se observó una diferencia en cuanto a género, predominó el masculino con $58 \%$ y el femenino con $42 \%$, que coinciden con los datos de fallecimientos en México; mientras que en el estudio realizado por Escalona Leyva y colaboradores también se muestra similitud respecto al género con nuestro reporte, pues señalaron como parte del perfil epidemiológico de su población donante un predominio en el sexo masculino con $60.67 \%$.

Tras relacionar las edades de los donantes con el resultado de la densidad celular, fueron varios los casos en los que se pudo apreciar que la edad no garantiza la calidad celular de los botones corneales, ya que la densidad depende de más factores implicados y serán los que definirán la calidad final de los tejidos y su viabilidad para fines ópticos.

La edad media considerada en nuestra muestra fue de 58 años, coincidiendo con otros estudios que también refuerzan la importancia de la morfología celular más que la edad, como en el caso del estudio epidemiológico realizado en 2015 en el Hospital Universitário Onofre Lopes de la Universidad Federal de Río Grande del Norte, Brasil, donde la edad promedio de los donantes fue de 49 años, además de que la mitad de los pacientes que lograron ser trasplantados poseía edad de hasta 52 años. Esto nos deja ver una notable diferencia de casi una década del rango etario entre su estudio y el nuestro.

En cuanto a las comorbilidades, puntualizamos que el $48 \%$ de la muestra total de donadores padecía hipertensión arterial sistémica, cifra que concuerda con la distribución de casos en el panorama epide- 
miológico de los mexicanos, donde se reporta una cifra en aumento constante desde el año 2010 hasta el año 2019, manteniéndose como la novena causa de defunción y principal factor de riesgo de muertes prevenibles. Sin embargo, en otro estudio realizado en Coahuila en 2014 por Guadalupe Contreras y su equipo, se menciona que el mayor porcentaje de presentación fue padecer diabetes mellitus tipo 2 con un $60 \%$, dejando en segundo lugar en frecuencia la hipertensión arterial sistémica sólo en $23.3 \%$ de su muestra, esa diferencia de seis años ha modificado las estadísticas actuales con predominio en la población hipertensa.

En nuestra investigación encontramos que el evento cerebral vascular fue el diagnóstico de defunción, responsable de la muerte del $31 \%$ de los donantes, situación que también continúa siendo frecuente en nuestro país, como lo mencionó Sergio de la Garza y su grupo en 2018 en su estudio realizado en el Hospital General de Matamoros, donde en una población de 4,619 pacientes ingresados a dicho nosocomio, 438 tuvieron como diagnóstico de defunción EVC, es decir, el $9.4 \%$. En otro estudio descriptivo y transversal realizado en el Banco de Ojos del Instituto Cubano de Oftalmología se recibieron 590 donantes y establecieron que las principales causas de muerte fueron la enfermedad cardiovascular (29\%) y las cerebrovasculares $(24.7 \%)$. Deja en segundo lugar a nuestros resultados. ${ }^{11,12}$

Referente al destino final de los 61 tejidos que fueron recibidos y analizados, no todos pudieron ser trasplantados para finalidad óptica debido a las condiciones celulares encontradas tras la realización de la microscopia especular, por lo que se determinó que el $18 \%$ de esos tejidos tuvo como destino final su envío al Departamento de Patología, mientras que el $82 \%$ pudo ser trasplantado con fines ópticos, mejorando así la calidad de vida de los pacientes que se encontraban en lista de espera para trasplante corneal. Si comparamos los datos de nuestro estudio con los reportados en el Instituto Cubano de Oftalmología, en donde el trasplante terapéutico y/o tectónico se realizó en un porcentaje elevado, la mayoría de los trasplantes realizados tuvieron finalidad óptica al igual que nosotros con 207 casos (76.6\%). Mientras que la finalidad terapéutica y/o tectónica estuvo representada en 63 casos de trasplante (23.3\%). Por lo que podemos deducir que tanto en nuestro medio, así como en otros países, la realización de trasplantes con finalidad óptica es la que predomina. ${ }^{13}$

\section{CONCLUSIONES}

Estudios epidemiológicos han sido realizados con el objetivo de trazar el perfil de los donantes de órganos y tejidos, para realizar el trasplante de córnea; así como estudios de pacientes trasplantados en diferentes espacios demográficos, que han permitido la comparación y análisis de las variables determinantes para los más diversos resultados vinculados al trasplante corneal.

Con este estudio logramos concluir que el perfil epidemiológico que caracterizó al donante de tejido corneal en Banco de Tejido Ocular del Hospital General de Cholula fue donante perteneciente al género masculino, finado por un evento cerebral vascular, entre 50 y 80 años con hipertensión arterial sistémica como comorbilidad principal.

Del total de la muestra considerada para esta investigación, de los 61 tejidos analizados en su mayoría contaron con las características y condiciones adecuadas para cumplir la finalidad óptica. Con ello reforzamos que el conocimiento del perfil clínico y epidemiológico de los donantes de córnea con fines de trasplante puede posibilitar la identificación de grupos de riesgo para fines de prevención e implementación de cuidados que resulten en mejores pronósticos.

\section{REFERENCIAS}

1. Rojas Dosal JA. Simposio trasplante de córnea. Rev Hosp Jua Mex. 2000; 67: 139-143.

2. González-Pérez MK, Neri-Vela R, Quintero-Castañón R. El trasplante de córnea en México. Antecedentes históricos. Rev Mex Oftalmol. 2012; 86 (4): 187-190.

3. Centro Nacional de Donación y Trasplantes. Reporte anual de donación y trasplante México. México: Sistema Informático del Registro Nacional de Trasplantes; 2020. pp. 1-81.

4. Area Crespo A. Criopreservación y vitrificación de córneas humanas. España: Universidad de Coruña; 2016.

5. Verdiguel Sotelo K, Carrasco Quiroz A, López Macías PA. El trasplante de calidad de tejido corneal procurado por personal médico no oftalmólogo capacitado córnea en México. Antecedentes históricos. Rev Med Inst Mex Seguro Soc [Internet]. 2016 [citado 7 de julio de 2019]; 54 (6): 688-695. Disponible en: https://www.medigraphic.com/pdfs/imss/im2016/im166c.pdf

6. Tejeda Mondragón M. Manual de procedimientos para el establecimiento y la operación del Banco de Ojos en el Hospital General de Cholula. Puebla; 2020.

7. Observatorio Mexicano de Enfermedades No Transmisibles (OMENT). Panorama epidemiológico 2018. Enfermedades no transmisibles [Internet]. México: 2018. pp. 9-92. [citado 26 de julio de 2019] Disponible en: gob.mx/salud/documentos/panoOMENT/Panorama_OMENT_2018.pdf

8. De la Garza Longori RS, Maldonado Mancillas JA. Incidencia de enfermedad cerebrovascular en un servicio de Medicina Interna. Med Int Méx [Internet]. 2018 [citado 17 agosto 2019]; 
34 (6): 874-880. Disponible en: https://doi.org/10.24245/mim. v34i6.2062

9. ClE-10, Ministerio de sanidad, servicios sociales e igualdad. Versión 1.0. 2015.

10. Pérez-Padilla JR. Muertes respiratorias en México, 2015. Neumol Cir Torax. 2018; 77 (3): 198-202.

11. Estadísticas de mortalidad. INEGI. México: 2018.

12. Campos-Nonato I, Hernández-Barrera L, Pedroza-Tobías A, Medina C, Barquera S. Hipertensión arterial en adultos mexicanos: prevalencia, diagnóstico y tipo de tratamiento. Ensanut MC 2016. Salud pública Méx. 2018; 60 (3): 233-243.
13. Escalona LET, Pérez PZ, Castillo PA, Jareño OM, López HSM. Perfil epidemiológico del donante y del receptor en trasplante de córnea en el Instituto Cubano de Oftalmología "Ramón Pando Ferrer". Rev Cub Oftal [Internet]. 2014 [citado 19 de julio de 2019]; 27 (4): 558-568. Disponible en: http://scielo.sld.cu/pdf/oft/ v27n4/oft06414.pdf

Correspondencia:

Guadalupe Merino-Cabrera

E-mail: lupmer_cab@outlook.com 